\title{
AUTONOMIA DE PESSOAS COM DEFICIÊNCIA RESIDENTES EM UBERLÂNDIA: UMA APLICAÇÃO DO MODELO GRADE OF MEMBERSHIP
}

\author{
AUTONOMY OF PEOPLE WITH DISABILITIES RESIDENTS IN UBERLÂN- \\ DIA: AN APPLICATION OF THE GRADE OF MEMBERSHIP MODEL
}

\author{
Francismara Fernandes Guerra ${ }^{1}$ e Luiz Bertolucci ${ }^{2}$
}

Recebido em: 24/06/2016

Aprovado em: 01/12/2016

\section{RESUMO}

Propõe-se, neste trabalho, estabelecer um escore de autonomia das pessoas com deficiência residentes em Uberlândia, Minas Gerais, acompanhadas em 2004 e 2008, a fim de verificar se, nesse período, houve ou não um ganho de autonomia. Para proceder à avaliação, foi utilizado um conjunto de variáveis selecionadas a partir dos dados dos Levantamentos de Informações Econômico-Sociais da População com Deficiência no Município de Uberlândia, Minas Gerais. Já para determinar o escore da autonomia, utilizou-se o Grade of Membership. Observou-se que o ritmo de ganho de autonomia dessa população é lento e que não houve uma melhoria generalizada da autonomia da população com deficiência nesse período. Acredita-se que esse resultado possa também estar relacionado com o curto prazo de quatro anos de acompanhamento, insuficiente para promover o aumento da autonomia das pessoas com deficiência, ou com as políticas sociais empreendidas nesse sentido, que podem não ter sido corretamente focalizadas.

Palavras-chave: Deficiência; Autonomia; Grade of Membership.

\begin{abstract}
It is proposed in this paper to establish a score of autonomy of disabled residents in Uberlândia, Minas Gerais, followed in 2004 and 2008 in order to verify that in this period there was a gain of autonomy of them. The evaluation used a set of selected variables from the data of Economic and Social Information Surveys Population with disabilities in the city of Uberlandia, Minas Gerais. And to determine the score of autonomy, we used the Grade of Membership. It was observed that the pace of the disabled autonomy gain is slow and that there was no overall improvement of the autonomy of disabled population in this period. It is believed that this result can also be related to the short period of four years follow-up, insufficient to promote increased autonomy of the disabled; or that social policies undertaken in this respect were not correctly focused. Keywords: Disability; Autonomy; Grade of Membership.
\end{abstract}

1 Possui graduação em Licenciatura em Matemática pela Universidade Federal de Ouro Preto - UFOP (2006), Mestrado (2010) e Doutorado (2014) em Demografia pelo Centro de Desenvolvimento e Planejamento Regional - CEDEPLAR da Universidade Federal de Minas Gerais - UFMG (2010) e Pós-doutorado na área de Economia Doméstica pela Universidade Federal de Viçosa - UFV (2015). Atualmente realiza o segundo pós-doutorado também na área de Economia Doméstica pela Universidade Federal de Viçosa - UFV.

2 Doutor em Demografia pelo Centro de Desenvolvimento e Planejamento Regional CEDEPLAR da Universidade Federal de Minas Gerais. Graduação em Ciências Econômicas pela Universidade Federal de Uberlândia - UFU. Atualmente é servidor da UFU, ocupando o cargo de Diretor de Provimento, Acompanhamento e Administração de Carreiras. Pertence ao quadro de pesquisadores do Centro de Pesquisas Econômico-Sociais - CEPES do Instituto de Economia. 


\section{Introdução}

Como resultado da preocupação do governo federal em especificar e detalhar as dimensões da deficiência no Brasil, elaborou-se o Censo Demográfico de 2000. Com relação às deficiências de natureza sensorial ou física, o novo questionário inquiriu sobre as capacidades de enxergar, ouvir e caminhar/subir escadas, que vão desde a opção incapaz até a de nenhuma dificuldade, passando pelas opções de grande dificuldade permanente ou alguma dificuldade permanente. Por outro lado, tais mudanças não possibilitam verificar a temporalidade da deficiência e sua possibilidade de recuperação, visto que alguns quesitos se restringem a perguntas em torno da deficiência permanente, como, por exemplo, da deficiência mental.

Assim, as informações coletadas pelo Censo de 2000, apesar de representarem significativo avanço no conhecimento da população com deficiência, não permitem estudar, com maior detalhamento, avanços na autonomia desse contingente populacional. Para tanto, seria necessária a inclusão de outras variáveis que detalhassem a situação de suporte familiar e institucional, de adaptação domiciliar quanto à deficiência, de motivos de migração e da não inclusão no mercado de trabalho, o momento de aquisição e a causa da deficiência, dentre outras características dessas pessoas que não puderam ser captadas pelas pesquisas censitárias.

Com as informações censitárias enumerando o expressivo contingente de pessoas com deficiência, os tomadores de decisão passam a demandar conhecimentos complementares e específicos, a fim de decidirem sobre políticas públicas, gestões de recursos humanos, dentre outras iniciativas que possam ser ajustadas ao relevante aparato legal definido, ampliado e em vias de consolidação desde a Lei Orgânica de Assistência Social (LOAS) de 1993.
Para tanto, na última década, em busca de maior detalhamento de quesitos sobre indivíduos com deficiência, principalmente em nível municipal, diversas pesquisas foram realizadas: algumas focadas na obtenção de informações médicas e de saúde (Cury, 2004; Corde, 2004; César et. al, 2005), e outras com enfoque socioeconômico buscando verificar, entre outras questões, a inclusão da pessoas com deficiência no sistema educacional ou no mercado de trabalho (Valdés, 200?; Gomes e Barbosa, 2006; Camargos Borges, 2005; Ferreira et. al, 2009).

Nesse sentido, este trabalho se propõe a estudar um quesito pouco discutido na literatura, a autonomia dos deficientes. Por meio de pesquisas realizadas na cidade de Uberlândia, Minas Gerais, um centro urbano que conta com ampla rede de assistência, apoio e reabilitação de pessoas com deficiência, aqui denominadas Levantamento de Informações das Pessoas com Deficiência (LIPD), é possível verificar se, no período entre 2004 e 2008, os entrevistados tiveram ganhos de autonomia. Uma vez constatados tais ganhos, poderemos atribuí-los à inserção de pessoas com deficiência no mercado de trabalho, do acesso à educação, dos benefícios sociais, dos aparelhos de reabilitação, dos domicílios adaptados, do suporte institucional e familiar, dentre outros quesitos, proporcionados por políticas públicas nesse período.

\section{Referencial teórico}

A população de pessoas com deficiência tem sido alvo de discussão desde o advento da Declaração de Direitos Humanos, em 1948, quando as Nações Unidas definiram, em seu artigo 25, que

todo ser humano tem direito a um padrão de vida capaz de assegurar-lhe, e a sua família, saúde e bem-estar, inclusive alimentação, vestuário, habitação, cuidados médicos e os servi- 
ços sociais indispensáveis, e direito à segurança em caso de desemprego, doença, invalidez, viuvez, velhice ou outros casos de perda dos meios de subsistência em circunstâncias fora de seu controle (ONU, 1948, p.?).

A partir do termo invalidez, passou-se a considerar um conceito mais amplo de deficiência, a fim de atender à busca por autonomia do expressivo contingente de população com deficiência adquirida nas duas últimas grandes guerras, bem como dos deficientes congênitos por conta de doenças, fome, contaminações diversas, acidentes ou violência sofrida pela mãe no período gestacional, criando-se um ambiente cultural de fortes discussões médico-sociais que vão culminar, em 1971, com a Declaração dos Direitos do Deficiente Mental, pela Organização das Nações Unidas (ONU). O mundo reconhece, de maneira formal, a presença dos deficientes no contexto social, passando a envidar esforços, por meio de políticas e ações focalizadas, para que essas pessoas sejam incluídas e beneficiadas pela educação, tendo oportunidade de trabalho, apoio legal e garantias de apoio familiar ou institucional (ONU, 1971).

Os debates sobre o tema avançam na década de 70 , culminando com a Declaração dos Direitos das Pessoas Deficientes, aprovada pela Assembleia Geral da ONU, em dezembro de 1975 , que afirma em seu artigo $1^{\text {o: }}$ " $O$ termo 'pessoas deficientes' refere-se a qualquer pessoa incapaz de assegurar por si mesma, total ou parcialmente, as necessidades de uma vida individual ou social normal, em decorrência de uma deficiência congênita ou não, e suas capacidades físicas ou mentais" (ONU, 1975, p.?).

Passa-se, portanto, a contrapor-se os conceitos médico e social de deficiência (Omote, 1994; Fernald et. al, 1996; Amiralian et.al, 2000). Para além das deficiências físicas ou sensoriais, considera-se, no modelo social, que o deficiente terá sua autonomia comprometida, ou seja, que experimenta a incapacidade de garantir suas necessidades individuais, justamente por não estar incluído na sociedade. Sua exclusão, representada pela escassez de recursos que minimizariam ou normalizariam a deficiência, mostra que essa pessoa não consegue superar as barreiras socioeconômicas e culturais que lhe garantiriam certa independência (Manton, 1997; Miles, 2000).

A autonomia do deficiente, ou inclusão ótima, seria considerada o acesso aos recursos da comunidade: ambientes físicos (moradia), sociais e culturais, transporte, informação, tecnologia, meios de comunicação, educação, justiça, serviço público, emprego, esporte e recreação, votação e oração. Reconhece-se que essa autonomia conseguida por poucos deve ser proporcionada a muitos e que, para tanto, deve-se eliminar as barreiras ambientais, eletrônicas e atitudinais que impedem a plena inclusão dos deficientes na vida comunitária (RI, 1999).

O ano de 1981 foi proclamado pelas Nações Unidas como o Ano Internacional das Pessoas Deficientes. Intensificam-se, então, os movimentos internacionais, com impactos relevantes no âmbito das nações signatárias, para a inclusão das pessoas com deficiência.

No Brasil, os diversos acontecimentos de natureza política ou assistencialista acabaram por motivar o movimento das pessoas com deficiência, fazendo com que o mesmo ganhasse novo rumo em suas reivindicações, culminando com resoluções importantes criadas a partir da promulgação da Constituição Brasileira de 1988. As pessoas com deficiência tiveram na Constituição Federal o aparato legal que assegura o Estado de Direito, em que o deficiente encontra na Lei a possibilidade de viver e conviver socialmente, superando limites, barreiras e agressões e sobressaindose como pessoa, sujeito com deficiência, mas também com possibilidades inatas ou conquistas que não devem ser ofuscadas pela deficiência (Silva, 2002). No ano seguinte, a Lei n. ${ }^{\text { }} 7853 / 1989$, que 
dispõe sobre o apoio às pessoas portadoras de deficiência e sua integração social, determina que sejam incluídas no censo demográfico de 1990 e nos subsequentes questões concernentes à problemática da pessoa com deficiência.

Nesse contexto, para conhecer a população residente no país com deficiência, acentuam-se os estudos no Brasil sobre a temática, seja pelas mudanças no aparato legal que estabelece os direitos dessa parcela populacional (Valdés, 200?; Ferreira et. al, 2009), seja pela contribuição de pesquisas em nível censitário e de abrangência nacional, principalmente dos Censos Demográficos de 1991 e 2000, realizados pelo Instituto Brasileiro de Geografia e Estatística (IBGE), bem como de outras fontes de informação com possibilidades de análise em torno de saúde, educação, inserção no mercado de trabalho, dentre outras questões (Datasus - Ministério da Saúde; Rais-Migra, base de dados do Ministério do Trabalho).

O Censo Demográfico de 1991 já havia apresentado uma estimativa de 1,7 milhão de pessoas com deficiência no Brasil, aproximadamente 1,2\% da população total. Essa proporção de deficientes diferiu das estimativas da ONU (10\%); da Pesquisa Nacional por Amostra de Domicílios (PNAD) realizada pelo IBGE em 1981, que estimou 1,8\%; da Pesquisa Nacional de Saúde, realizada em 1989 pelo IBGE, que estimou $8,9 \%$ de pessoas com deficiência (Chagas, 1991). Diante desses diferentes resultados, o Censo Demográfico de 2000 contou com remodelação dos quesitos que sondam a deficiência, baseando-se em questões propostas pelas Nações Unidas, a partir da sugestão do Grupo de Washington, composto de especialistas sobre o tema deficiência, de diferentes países, visando, inclusive, à harmonização dos censos demográficos no mundo para comparações internacionais posteriores (WG, 2010).

Assim, os resultados da Tabulação Avançada do Censo Demográfico brasileiro de 2000 indicaram que aproximadamente 24,5 milhões de pessoas, ou seja, $14,5 \%$ da população total, apresentaram algum tipo de incapacidade ou deficiência. Incluem-se nessa categoria as pessoas com ao menos alguma dificuldade de enxergar, ouvir e locomover-se ou com alguma deficiência física ou mental (SICORDE, 2009). Isso indica que considerável parcela da população apresenta algum tipo de deficiência e, consequentemente, necessita de alguma assistência especializada para o pleno desenvolvimento de sua autonomia e para uma participação mais igualitária na sociedade. Portanto, ainda que esses dados se configurem como uma fonte potencial de informações para as políticas públicas, apenas mostram a descrição do cenário atual da deficiência no Brasil, sugerindo, com isso, que pesquisas complementares são necessárias para proporcionar os meios de promover a autonomia da pessoa com deficiência.

\section{Método de pesquisa}

Neste trabalho, empregam-se os dados dos LIPDs, que permitem obter informações de natureza longitudinal de indivíduos com deficiência residentes em Uberlândia, Minas Gerais, associando-se mudanças socioeconômicas a mudanças de autonomia, por tipo de deficiência. Para tanto, foram analisadas 1.063 entrevistas de pessoas observadas em 2004 e que não morreram ou migraram até 2008.

A fim de estabelecer um escore de autonomia dos deficientes, utilizou-se o Grade of Membership (GoM) (Guedes, Saviero, Machado, 2010; Guedes et.al, 2010; Caetano e Machado, 2009). O GoM é um modelo matemático conhecido por gerar perfis extremos, característicos de uma população ou parte dela, e por fornecer os graus de pertencimento de cada indivíduo pesquisado em relação a esses perfis extremos.

Em um primeiro momento, induziu-se o GoM a gerar dois perfis extre- 
mos, com base nas variáveis pesquisadas (socioeconômicas e demográficas com informação sobre mobilidade espacial), que descrevessem perfis realmente extremos/antagônicos, os quais foram denominados de Perfil Extremo Ótimo e de Perfil Extremo Precário (Quadro 1). Em seguida, o GoM indica o grau de pertencimento (g), que determina a proximidade dos indivíduos com cada um desses perfis. Vale lembrar que nessas condições, como o grau de pertencimento de um perfil é sempre complementar ao do outro perfil, vê-se que, independente do g que se escolha analisar, este sempre dará uma coordenada de posicionamento entre duas situações opostas. Essa coordenada é então usada como um escore de autonomia da pessoa com deficiência.

Os perfis extremos predefinidos são idênticos em 2004 e 2008, como apresentado no Quadro 1, em que se descreve qual seria um perfil extremamente precário e extremamente ótimo. Desse modo, em um segundo momento, é possível comparar longitudinalmente a mudança da autonomia das pessoas com deficiência, no contexto analisado, por meio dos graus de pertencimento dos perfis extremos, denominados de Perfil Extremo Ótimo e de Perfil Extremo Precário.

Quadro 1- Descrição dos perfis extremos das pessoas com deficiência

\begin{tabular}{|c|c|c|}
\hline Variável & Perfil Extremo Ótimo & Perfil Extremo Precário \\
\hline $\begin{array}{c}\text { Material de construção } \\
\text { do domicílio }\end{array}$ & Alvenaria & Outro \\
\hline Situação de residência & Financiada/própria & Alugada/cedida \\
\hline Sexo & Masculino & Feminino \\
\hline Grupo etário & 25 a 49 anos & 24 anos ou menos \\
\hline $\begin{array}{l}\text { Domicílio possui adap- } \\
\text { tação }\end{array}$ & Sim & Não \\
\hline $\begin{array}{c}\text { Relação com o respon- } \\
\text { sável pelo domicílio }\end{array}$ & Responsável & Filho/enteado \\
\hline Migração & Não migrante & Migrante \\
\hline Estado civil & Casado & Solteiro \\
\hline Filhos & Não tem filhos & Tem filho(s) com deficiência \\
\hline Raça/cor & Branca & Preta/negra \\
\hline Frequenta associação & Sim & Não \\
\hline Escolaridade & $\begin{array}{l}\text { Ensino superior completo ou } \\
\text { pós-graduação }\end{array}$ & Analfabeto \\
\hline $\begin{array}{l}\text { Fez curso técnico ou } \\
\text { profissionalizante }\end{array}$ & Sim & Não \\
\hline $\begin{array}{l}\text { Quando adquiriu a } \\
\text { deficiência }\end{array}$ & Após o nascimento & Desde o nascimento \\
\hline $\begin{array}{c}\text { Precisa de ajuda } \mathrm{p} / \text { sair } \\
\text { de casa }\end{array}$ & Não precisa de ajuda & Precisa, mas não tem ajuda \\
\hline Recebe algum benefício & Sim & Não \\
\hline Situação do trabalho & $\begin{array}{l}\text { Empregado com carteira, funcio- } \\
\text { nário público ou empregador }\end{array}$ & Não trabalha \\
\hline Rendimento individual & Mais de um salário mínimo & Até um salário mínimo \\
\hline $\begin{array}{l}\text { Necessita e/ou possui } \\
\text { aparelho }\end{array}$ & Não necessita & Necessita, mas não possui \\
\hline
\end{tabular}

Notas: 1) todas as variáveis são categóricas e podem possuir mais de duas categorias; 2) as variáveis sexo, migração, raça/cor e quando adquiriu a deficiência são consideradas fixas, segundo as informações de 2004, para maior consistência dos resultados.

Fonte: elaboração própria. 
Para efeito das análises descritivas, optou-se por interpretar exclusivamente os g's do Perfil Ótimo. Isto é, quanto mais próximo o $\mathrm{g}$ do indivíduo estiver de um, maior será a autonomia do indivíduo, e, quanto maior for a diferença entre os escores de 2008 e 2004 , maior será o ganho de autonomia socioeconômica da pessoa com deficiência, no período analisado.

\section{Apresentação e discussão dos resul- tados}

Pela Tabela 1, pode-se inferir que, mesmo sendo uma pequena mudança, houve um ganho da autonomia da população com deficiência como um todo. Mas, estratificando-se por tipo de deficiência, somente o grupo de deficientes auditivos e visuais, em específico, percebeu melhorias reais. Para os demais tipos de deficiência, no entanto, não se pode garantir que houve mudança da autonomia, seja positiva ou negativa.

Ressalta-se, ainda, que houve uma redução da heterogeneidade intragrupos, de modo que, apesar da grande variabilidade de níveis de autonomia dentro de cada grupo de deficiência, é possível verificar que as diferenças socioeconômicas e demográficas reduziram. A única exceção a esse comportamento é o caso dos deficientes mentais, cuja variabilidade aumentou.

Para qualquer grupo de deficiência, não se observou uma aproximação razoável do grau de pertencimento ao Perfil Extremo Ótimo, que seria de um, sugerindo uma situação bem aquém daquela descrita no próprio perfil. A melhor situação observada foi a dos deficientes físicos, embora não se possa dizer que tenham sido mais privilegiados do que os demais, visto que a diferença é mínima.

Tabela 1 - Média dos graus de pertencimento ao Perfil Ótimo segundo o tipo de deficiência, 2004 e 2008

\begin{tabular}{ccc}
\hline Tipo de deficiência & 2004 & 2008 \\
\hline Múltipla & 0,5697 & 0,5835 \\
& $(0,123)$ & $(0,120)$ \\
Auditiva* & 0,5779 & 0,6001 \\
& $(0,126)$ & $(0,116)$ \\
Física & 0,6257 & 0,6249 \\
& $(0,127)$ & $(0,115)$ \\
Mental & 0,5208 & 0,5244 \\
& $(0,085)$ & $(0,093)$ \\
Visual** & 0,6040 & 0,6197 \\
& $(0,121)$ & $(0,116)$ \\
Total** & 0,5918 & 0,5983 \\
& $(0,126)$ & $(0,118)$ \\
\hline
\end{tabular}

Nota: 1) o tipo de deficiência corresponde àquele declarado em 2004; 2) desvio padrão entre parênteses; 3) diferenças significativas ao nível de “*” $1 \% \mathrm{e}$ “**” $5 \%$.

Fonte: LIPD 2004 e 2008. 
Quanto aos resultados apresentados na Tabela 2, define-se um quadro ambíguo em relação às mudanças no status da autonomia individual. Podese dizer que uma parcela expressiva das pessoas com deficiência, tanto total quanto estratificada por deficiência, experimentou um ganho na autonomia, que pode estar associada a uma melho- ria das condições de vida. Em contrapartida, pelo menos uma em cada dez pessoas com deficiência se manteve em uma situação igual àquela da condição inicial. Verifica-se, também, que uma elevada razão das pessoas com deficiência, aproximadamente quatro em cada dez, sofreu uma regressão no seu status de autonomia de 2004 para 2008.

Tabela 2 - Proporção da população com deficiência por tipo de deficiência segundo a mudança no status da autonomia entre 2004 e $2008(\%)$

\begin{tabular}{cccc}
\hline Tipo de deficiência & Melhora & Estabilidade & Piora \\
\hline Múltipla $(\mathrm{n}=114)$ & 50,0 & 12,3 & 37,7 \\
Auditiva $(\mathrm{n}=124)$ & 57,3 & 12,9 & 29,8 \\
Física $(\mathrm{n}=477)$ & 42,6 & 12,2 & 45,3 \\
Mental $(\mathrm{n}=188)$ & 42,6 & 18,1 & 39,4 \\
Visual $(\mathrm{n}=153)$ & 49,7 & 13,1 & 37,3 \\
Total $(\mathrm{n}=1063)$ & 46,1 & 13,5 & 40,5 \\
\hline
\end{tabular}

Nota: 1) o tipo de deficiência corresponde àquele declarado em 2004; 2) no total da amostra estão inclusos também outra deficiência e deficiência não declarada.

Fonte: LIPD 2004 e 2008.

Dentre os escores individuais calculados, os escores mínimos encontrados foram de 0,2000 e 0,2353 em 2004 e 2008, respectivamente, indicando que a autonomia dos indivíduos em situação mais precária progrediu. Em contrapartida, o fato de o escore máximo encontrado ter caído de 0,9333 para 0,9286 , no mesmo período, leva a crer que os indivíduos mais próximos de uma situação ótima encontram dificuldades de progredir.

Observa-se que a pessoa com deficiência em pior situação, ranqueada pelo escore mais baixo, está mais distante do Perfil Extremo Precário do que a pessoa com deficiência em melhor situação - escore mais elevado - em comparação ao Perfil Extremo Ótimo. Mas isso não quer dizer que a pessoa com deficiência em geral se encontre em uma situação favorável, até mesmo porque a amostra não inclui indivíduos sem deficiência, o que dificulta a comparação entre o status de autonomia entre pessoas com e sem deficiência.
Visto que não há representantes puros $^{3}$ dos perfis extremos e que há uma grande heterogeneidade dentro da população com deficiência, na Tabela 3, apresentam-se cinco tipos de situações ou de perfis mistos/medianos ordenados pelos escores dos indivíduos. A ordenação desses perfis parte de uma situação de autonomia menos favorável, de acordo com os mesmos critérios utilizados anteriormente, a uma situação mais favorável.

Embora se possa perceber que houve uma redução na proporção da população nas piores situações, precária e regular, verifica-se também uma queda proporcional na melhor situação, ótima, quanto à autonomia. Esse deslocamento ocorreu, provavelmente, na direção das situações medianas, intermediária e boa, em que grande parte das pessoas com deficiência se encontra. 
Tabela 3 - Proporção da população com deficiência segundo a situação de autonomia, 2004 e $2008(\%)$

\begin{tabular}{ccc}
\hline Situação & $\% 2004$ & $\% 2008$ \\
\hline Precária $(\mathrm{g} \leq 0,25)$ & 0,20 & 0,1 \\
Regular $(0,25<\mathrm{g} \leq 0,40)$ & 6,2 & 4,6 \\
Intermediária $(0,40<\mathrm{g} \leq 0,60)$ & 50,6 & 48,7 \\
Boa $(0,60<\mathrm{g} \leq 0,75)$ & 32,6 & 37,4 \\
Ótima $(\mathrm{g}>0,75)$ & 10,3 & 9,1
\end{tabular}

Fonte: LIPD 2004 e 2008

Ainda que os resultados obtidos pareçam contraditórios, pode-se inferir que houve uma pequena mudança da autonomia dos deficientes. Por esses resultados, não se pode dizer que ocorreu uma melhoria generalizada da autonomia nesse curto espaço de tempo, já que o deslocamento de contingente populacional ocorreu nos dois sentidos, de cima para baixo e de baixo para cima, que os fluxos em sentidos contrários acabam compensando parcialmente o ganho ou a perda de contingente populacional da situação inicial.

A causa dessa ambiguidade nos resultados provém das mudanças relatadas pelos entrevistados. A redução do número de domicílios de alvenaria e de indivíduos que frequentam algum tipo de associação beneficente, assim como o aumento de filhos com deficiência e a queda da renda per capita, pesou negativamente sobre a autonomia das pessoas com deficiência. Por outro lado, o crescimento da proporção de deficientes que são responsáveis pelo domicílio e que fizeram algum curso técnico ou profissionalizante e superior, bem como a redução de analfabetos, a melhoria na obtenção de aparelhos especializados, a ajuda para locomoção, os benefícios governamentais e as melhores oportunidades de trabalho, implicou positivamente a autonomia das pessoas com deficiência.

Há, por fim, situações de certa maneira compensatórias. Uma delas refere-se aos indivíduos que não responderam a determinadas questões na primeira onda da pesquisa e o fizeram na segunda, o que levou a um aumento na proporção de residências tanto alugadas/cedidas quanto próprias/financiadas e de domicílios com e sem adaptação. E a outra se refere a mudanças demográficas, como a redução de solteiros e de jovens, categorias que, conforme discriminado do Quadro 1, afetam negativamente a autonomia das pessoas com deficiência.

\section{Considerações finais}

Com o emprego do GoM, foi possível estabelecer um ranking que determinou a posição dos indivíduos com deficiência entre uma situação precária e uma situação melhor, denominada, neste trabalho, de ótima. Esse ranking, por sua vez, possibilitou calcular o ganho de autonomia das pessoas com deficiência. O uso do GoM destaca-se, ainda, por incorporar as características empíricas e as informações disponíveis da população, bem como por poder ser empregado com o intuito de estabelecer prioridade de atendimento em quaisquer circunstâncias, como foi o caso deste estudo, ao verificar a precariedade das pessoas com deficiência.

Apesar de o arcabouço legal definir direitos explícitos a essa população vulnerável, há quase 35 anos, quando se declararam os direitos das pessoas com deficiência, e com maior ênfase aos brasileiros, desde a Lei Orgânica de Assistência Social, já em vigor por 17 anos, nota-se, com os resultados obtidos, que o ritmo de assunção dos direitos ou ganhos de autonomia é lento. Verifica-se que uma parte não desprezível dos en- 
trevistados conta com baixa autonomia social, pois são indivíduos com escolaridade e renda baixa, sem o aporte de recursos via benefícios sociais, sem a propriedade da moradia sem o necessário apoio institucional e familiar.

Este estudo revelou resultados positivos quanto à maior autonomia da pessoa com deficiência; no entanto, tal ganho não é generalizado, o que pode indicar baixa inclusão da pessoa com deficiência na sociedade. Acredita-se que esse resultado possa também estar relacionado com o curto prazo de quatro anos de acompanhamento, insuficiente para se promover o aumento da autonomia da pessoa com deficiência, ou ainda com as políticas sociais empreendidas nesse sentido, que podem não ter sido corretamente focalizadas.

\section{Referências}

AMIRALIAN, M. L. et.al. Conceituando deficiência. Rev. Saúde Pública, v. 34, n. 1, p. 97-103, 2000.

CAETANO, A. J.; MACHADO, C. J. Consistência e Identificabilidade no modelo Grade of Membership: uma nota metodológica. Revista Brasileira de Estudos de População, v. 26, n. 1, p. 145-149, 2009.

CAMARGOS BORGES, M. M. et. al. 2005. Levantamento de informações econômico-sociais das pessoas portadoras de deficiência no município de Uberlândia-MG. Relatório de Pesquisa. Uberlândia: CEPES/IEUFU. 107p. Disponível em: <http://www.ieufu.br/ cepes $>$.

CÉSAR, C.L.G; CARANDINA, L; ALVES, M.C.G.P; BARROS, M.B.A. ; GOLDBAUM M. 2005 Saúde e condição de vida em São Paulo - Inquérito Multicêntrico de Saúde no Estado de São Paulo - ISA-SP. 1a Ed. São Paulo: Annablume Editora.

CHAGAS, A. M. R. 1991. O porta- dor de deficiência no Brasil. Brasília: CORDE/IPEA. Série Estatística Especializada, v. 7, 38p.

CORDE - Coordenadoria Nacional para Integração da Pessoa Portadora de Deficiência. 2004. Relatório sobre a prevalência de deficiências, incapacidades e desvantagens. Niterói, Rio de Janeiro: CORDE - Ministério da Justiça.

CURY, G. C. C. 2004. Prevalência de Incapacidades em Sete Municípios do Estado de Minas Gerais. Belo Horizonte, CAADE/FUNDEP, Relatório de Pesquisa.

FERNALD, CD; ATKINSON, D; KEYNES, M ; HUTCHISON, T. 1996. Disability language preferences: towards some universal, culture free principles. In: 10TH WORLD CONGRESS OF THE INTERNATIONAL ASSOCIATION FOR THE SCIENTIFIC STUDY OF INTELLECTUAL DISABILITIES. Helsink. Anais... Helsink, p.61.

FERREIRA, E. W. et. al. 2009. Levantamento de informações econômicosociais das pessoas com deficiência no município de Uberlândia/MG - Fase II. Relatório de Pesquisa. Uberlândia: CEPES/IEUFU. 204p.

GUEDES, G. R. et. al. Identificabilidade e estabilidade dos parâmetros no método Grade of Membership (GoM): considerações metodológicas e práticas. Revista Brasileira de Estudos de População, 2010. (no prelo).

GUEDES, G. R.; SIVIERO, P. C.; MACHADO, C. J. 2010. Manual didático para o programa GoM 3.4: em busca de um modelo identificável e estável. Belo Horizonte: CEDEPLAR/UFMG.

MILES, S. 2000. Overcoming resource barriers. In INTERNATIONAL SPECIAL EDUCATION CONGRESS, 2000, Manchester. Anais... Manchester. Disponível em: <http://www. isec2000.org.uk ou em http://www. isec2000.org.uk/abstracts/papers_m/ miles_1.htm. Acesso e,: 03/04/2010. 
ORGANIZAÇÃO DAS NAÇÕES UNIDAS - ONU. Declaração Universal dos Direitos Humanos. Paris, 1948. Disponível em: $<\mathrm{http}: / /$ www.onu-brasil.org.br/documentos_direitoshumanos.php>. Acesso em: 11/11/2010.

Declaração dos Direitos do Deficiente Mental, 1971. Disponível em: http://www.cedipod.org.br/ w6dddm.htm. Acesso em: 24/06/2016.

Declaração dos Direitos das Pessoas Deficientes, 1975. Disponível em: http://portal.mec.gov.br/ seesp/arquivos/pdf/dec_def.pdf. Acesso em 24/06/2016.

OMOTE, S. Deficiência e não-deficiência: recortes do mesmo tecido. Revista Brasileira de Educação Especial, v.1, n.2, p.65-73; 1994.

RI, 1999. Carta para o Terceiro Milênio. Londres, Grã-Bretanha, Assembléia Governativa da Rehabilitation International. Disponível em http://portal. mj.gov.br/corde/carta_terceiro_mil.asp. Acesso em: 20/07/2010.

SICORDE, 2009. Resultados da Tabulação Avançada do Censo Demográfico 2000. Disponível em http://www. mj.gov.br/sedh/ct/corde/dpdh/sicorde/ censo2000.asp. Acesso em: 11/11/2010

SILVA, I. A. Construindo a cidadania: Uma análise introdutória sobre o direito à diferença. Uberlândia, 2002. Dissertação (Mestrado em História) Universidade Federal de Uberlândia.

VALDÉS, M. T. M. A Integração das Pessoas com Deficiência na Educação Superior no Brasil. 200? Disponível em: http://sid.usal.es/idocs/F8/ FDO12676/ integracion_educacion_superior_brasil. pdf, ou em http:// www2.iesalc.unesco. org.ve:2222/programas/Discapacitados/ Informe_Brasil\%20Discapacitados.pdf. Acesso em: 11/11/2010.

WG, 2010. Census Questions on Disability Endorsed by the Washing- ton Group. Disponível em http://www. cdc.gov/nchs/data/washington group/ WG_Short_Measure_on_Disability. pdf. Acesso em: 11/11/2010. 